\title{
THE MAXIMAL INVARIANCE GROUP OF NEWTON'S EQUATIONS FOR A FREE POINT PARTICLE ${ }^{\dagger}$
}

\author{
O. Jahn and V. V. Sreedhar \\ School of Theoretical Physics \\ Dublin Institute for Advanced Studies \\ 10, Burlington Road \\ Dublin 4, Ireland
}

\begin{abstract}
The maximal invariance group of Newton's equations for a free nonrelativistic point particle is shown to be larger than the Galilei group. It is a semi-direct product of the static (nine-parameter) Galilei group and an $S L(2, R)$ group containing timetranslations, dilations and a one-parameter group of time-dependent scalings called expansions. This group was first discovered by Niederer in the context of the free Schrödinger equation. We also provide a road map from the free nonrelativistic point particle to the equations of fluid mechanics to which the symmetry carries over. The hitherto unnoticed $S L(2, R)$ part of the symmetry group for fluid mechanics gives a theoretical explanation for an observed similarity between numerical simulations of supernova explosions and numerical simulations of experiments involving laser-induced implosions in inertial confinement plasmas. We also give examples of interacting many body systems of point particles which have this symmetry group.

$\dagger$ Dedicated to the memory of Prof. L. O'Raifeartaigh
\end{abstract}

DIAS-STP-01-02 
Almost all introductory books on the special theory of relativity mention, at least in passing, that Newton's equations of motion for a classical free nonrelativistic point particle are invariant under Galilei transformations. Probably not many eyebrows would be raised if we jumped from this fact to the conclusion that the Galilei transformations are the most general coordinate changes under which Newton's equations retain their form. It would therefore come as a considerable surprise to learn that there are other transformations which do the same. A simple example of such transformations may be given by noting that a freely moving point particle, with initial position $\mathbf{x}_{0}$ and velocity $\mathbf{v}_{0}$, traverses a straight line $\mathbf{x}(t)=\mathbf{x}_{0}+\mathbf{v}_{0} t$. This equation may be rewritten as $\mathbf{x} / t=\mathbf{v}_{0}+\mathbf{x}_{0} / t$. In this representation $t$ is replaced by $1 / t$, lengths are scaled by a factor proportional to time, and the initial position and velocity are interchanged; but the important point is that the trajectory remains a straight line. Hence, the new variables $\mathbf{x} / t, 1 / t$ satisfy the same equations as the old ones $\mathbf{x}, t[1]$. Therefore the following question naturally arises: What is the maximal invariance group of Newton's equations of motion for a classical free nonrelativistic point particle? The answer to this question reveals that the maximal invariance group is a twelve-parameter group consisting of the usual ten-parameter Galilei group, the one-parameter group of dilations, and a oneparameter group of time-dependent scalings called expansions which are nonrelativistic analogues of special conformal transformations. The existence of these transformations is not merely of academic interest. As explained in $[1,2]$, such transformations provide a theoretical explanation for the plausibility of simulating astrophysical systems like supernova explosions by performing laser-induced plasma implosions.

In order to find the maximal invariance group of an equation, one has to find the set of all spacetime transformations which leave it form-invariant up to a factor [3]. This condition is equivalent to the requirement that the Action be invariant. The Action $S$ for a free point particle of mass $m$, in $d$ space dimensions, at position $x_{i}(t)$ is given by 


$$
S=\frac{m}{2} \int d t\left(\frac{d x_{i}}{d t}\right)^{2}
$$

where $i=1 \cdots d$ and the sum over the index $i$ has been suppressed. Let us now consider a transformation to new coordinates $\xi, \tau$

$$
\xi_{i}=f_{i}(x, t), \quad \tau=h(x, t)
$$

We wish to find the most general functions $f_{i}, h$ which leave the Action (1) forminvariant:

$$
\int d t\left(\frac{d x_{i}}{d t}\right)^{2}=\int d \tau\left(\frac{d \xi_{i}}{d \tau}\right)^{2}
$$

This is achieved by requiring

$$
\frac{\left(\frac{\partial f_{i}}{\partial x_{j}} \frac{d x_{j}}{d t}+\frac{\partial f_{i}}{\partial t}\right)^{2}}{\left(\frac{\partial h}{\partial x_{k}} \frac{d x_{k}}{d t}+\frac{\partial h}{\partial t}\right)}=\left(\frac{d x_{i}}{d t}\right)^{2}+\frac{d}{d t} F(x, t)
$$

for arbitrary functions $x_{i}(t)$ where $F(x, t)$ is an arbitrary boundary term and

$$
\frac{d}{d t} F(x, t)=\left(\frac{\partial F}{\partial x_{i}} \frac{d x_{i}}{d t}+\frac{\partial F}{\partial t}\right)
$$

Comparing powers of $d x_{i} / d t$ on both sides of (4), we get

$$
\begin{aligned}
\frac{\partial h}{\partial x_{i}}=0 & \Rightarrow \quad \tau=h(t) \\
\left(\frac{\partial f_{i}}{\delta x_{j}}\right)\left(\frac{\partial f_{i}}{\delta x_{k}}\right) & =\frac{\partial h}{\partial t} \delta_{j k} \\
2 \frac{\partial f_{i}}{\partial x_{j}} \frac{\partial f_{i}}{\partial t} & =\frac{\partial F}{\partial x_{j}} \frac{\partial h}{\partial t} \\
\left(\frac{\partial f_{i}}{\partial t}\right)^{2} & =\frac{\partial F}{\partial t} \frac{\partial h}{\partial t}
\end{aligned}
$$

From (6) and (7) it follows that

$$
T_{l j k}+T_{l k j}=0 \quad \text { where } \quad T_{l j k}=\frac{\partial^{2} f_{i}}{\partial x_{l} \partial x_{j}} \frac{\partial f_{i}}{\partial x_{k}}
$$


Subtracting $T_{k j l}+T_{k l j}$ from the first equation in (10) and using the symmetry of $T_{l j k}$ under a permutation of the first two indices, it may be shown that $T_{l j k}$ is a totally symmetric tensor. Therefore $T_{i j k}=0$, which implies that $f_{i}$ is linear in $x$,

$$
f_{i}(x, t)=l(t) R_{i j}(t) x_{j}+m_{i}(t)
$$

It follows from (7) that $R$ can be chosen to be orthogonal, $R^{T}(t) R(t)=1$. Further, differentiating (8) with respect to $x_{k}$ and using the explicit expression for $f$ from (11) we get

$$
(l i) \delta_{k j}+l^{2}\left(R^{T} \dot{R}\right)_{k j}=\frac{1}{2} \frac{\partial F}{\partial x_{k} \partial x_{j}} \dot{h}
$$

where the dot refers to a derivative with respect to $t$. Since the right hand side and the first term on the left hand side are symmetric in $k$ and $j$, whereas the second term on the left hand side is antisymmetric, by virtue of being in the Lie algebra of the rotation group; we have $\dot{R}=0$ and thus, $R$ is a constant (rigid) rotation matrix. It then follows from (7) that

$$
\dot{h}=l^{2}
$$

Eliminating $F$ from (8) and (9) we get

$$
\frac{\partial^{2} f_{i}}{\partial t^{2}} \frac{\partial h}{\partial t}=\frac{\partial f_{i}}{\partial t} \frac{\partial^{2} h}{\partial t^{2}}
$$

Substituting (11) into this equation and comparing powers of $x$ we find

$$
\begin{gathered}
\ddot{l}=2(\dot{l})^{2} \\
l \ddot{m}_{i}=2\left(\dot{m}_{i} \dot{l}\right)
\end{gathered}
$$

Using (13), equation (15) can be rewritten in terms of $h(t)$ and takes the form

$$
\frac{\dddot{h}}{\grave{h}}-\frac{3}{2}\left(\frac{\ddot{h}}{\grave{h}}\right)^{2}=0
$$


The left hand side of the above equation is called the Schwarzian derivative of $h$ and a standard result of complex analysis - although only the real part is relevant here - is that the solution of the above differential equation is

$$
h(t)=\frac{\alpha t+\beta}{\gamma t+\delta} \quad \text { where } \quad \alpha \delta-\beta \gamma=1
$$

where $\alpha, \beta, \gamma, \delta$ are real. These transformations go by various names: fractional linear, projective, and global conformal. They form the group $S L(2, R)$. Substituting the above result in (13), and solving (16) for $m_{i}(t)$ we get

$$
l(t)=\frac{1}{\gamma t+\delta}, \quad m_{i}(t)=\frac{b_{i}}{\gamma t+\delta}+d_{i}
$$

where $b_{i}$ and $d_{i}$ are integration constants. From (11) and (18) we then have, for the most general transformations that leave Newton's equations invariant,

$$
\xi_{i}=\frac{R_{i j} x_{j}+a_{i}+v_{i} t}{\gamma t+\delta}, \quad \tau=\frac{\alpha t+\beta}{\gamma t+\delta} \quad \text { where } \quad \alpha \delta-\beta \gamma=1, \quad R^{T} R=1
$$

where $a_{i}$ and $v_{i}$ are constants expressible in terms of $b_{i}$ and $d_{i}$. It is useful to consider the following two special cases:

I. $\beta=\gamma=0, \alpha=\delta=1$ : Connected, Static Galilei Group G: In this case, we have

$$
g: \quad \tau=t, \quad \xi=R \mathbf{x}+\mathbf{a}+\mathbf{v} t
$$

These equations describe connected, static Galilei transformations which exclude parity and time-reversal. It is clear from (21) that this is a nine-parameter group.

II. $\mathbf{a}=\mathbf{v}=\mathbf{0}, R=1: S L(2, R)$ Transformations: In this case, we have

$$
\sigma: \quad \tau=\frac{\alpha t+\beta}{\gamma t+\delta}, \quad \xi=\frac{\mathbf{x}}{\gamma t+\delta} ; \quad \alpha \delta-\beta \gamma=1
$$

These are the $S L(2, R)$ generalisations of the inversion transformations presented in [1] and include time translations $(\gamma=0, \alpha=\delta=1)$, dilations $(\beta=\gamma=0)$ and a 
one-parameter group of time-dependent scalings called expansions $(\alpha=\delta=1, \beta=0)$. Since the parameters are constrained by the condition $\alpha \delta-\beta \gamma=1, S L(2, R)$ is a three-parameter group.

To understand the structure of the group, we study the relationship between the $S L(2, R)$ group and the connected static Galilei group $G$. Let us first consider a conjugation of a $g \in G$ by a $\sigma \in S L(2, R)$. By making three successive transformations of $x$ and $t$ we find that

$$
\sigma^{-1}(\alpha, \beta, \gamma, \delta) g(R, \mathbf{a}, \mathbf{v}) \sigma(\alpha, \beta, \gamma, \delta)=g\left(R, \mathbf{a}_{\sigma}, \mathbf{v}_{\sigma}\right)
$$

where

$$
\left(\begin{array}{l}
\mathbf{v}_{\sigma} \\
\mathbf{a}_{\sigma}
\end{array}\right)=\left(\begin{array}{ll}
\alpha & \gamma \\
\beta & \delta
\end{array}\right)\left(\begin{array}{l}
\mathbf{v} \\
\mathbf{a}
\end{array}\right)
$$

which shows that $G$ is an invariant subgroup. This result can be used to determine the product of two general elements $\sigma g$ and $\sigma^{\prime} g^{\prime}$ of the full group,

$$
\sigma g \sigma^{\prime} g^{\prime}=\sigma \sigma^{\prime} \tilde{g} g^{\prime}, \quad \text { where } \quad \tilde{g}=\sigma^{\prime-1} g \sigma^{\prime} \in G
$$

This shows that the full group is not a direct, but only a semi-direct product

$$
\mathcal{G}=S L(2, R) \wedge G
$$

As is apparent from (25), the two factors in the semi-direct product are on a different footing: while $G$ is an invariant subgroup, $S L(2, R)$ is not. Furthermore, recall that $G$ itself takes the form

$$
G=R \wedge(T \otimes B)
$$

where $R$ is the rotation group and $T$ and $B$ are translation and boost groups with parameters a and $\mathbf{v}$ respectively. Since $S L(2, R)$ commutes with $R, \mathcal{G}$ can be expressed as a single semi-direct product

$$
\mathcal{G}=(S L(2, R) \otimes R) \wedge(T \otimes B)
$$


Further it may be noted that the inversion $\Sigma$ considered in [1] is the special element of $S L(2, R)$ for which $(\alpha, \beta, \gamma, \delta)=(0,-1,1,0)$. Note that $\Sigma^{2}=P$ where $P$ is the parity transformation. This observation can be used to give a novel interpretation to a Galilei transformation. To see this we consider the coset elements $g_{\Sigma}(R, \mathbf{a}, \mathbf{v}) \equiv \Sigma g(R, \mathbf{a}, \mathbf{v})$, where $g \in G$, we have

$$
\begin{gathered}
g_{\Sigma}\left(R^{\prime}, \mathbf{a}^{\prime}, \mathbf{v}^{\prime}\right) g_{\Sigma}(R, \mathbf{a}, \mathbf{v})=g_{P}\left(R^{\prime} R, R^{\prime} \mathbf{a}-\mathbf{v}^{\prime}, R^{\prime} \mathbf{v}+\mathbf{a}^{\prime}\right) \\
\Rightarrow \quad g_{\Sigma}^{2}(R, \mathbf{a}, \mathbf{v})=g_{P}\left(R^{2}, R \mathbf{a}-\mathbf{v}, R \mathbf{v}+\mathbf{a}\right)
\end{gathered}
$$

where we have used the obvious notation $g_{P}(R, \mathbf{a}, \mathbf{v})=P g(R, \mathbf{a}, \mathbf{v})$. Since every pair of vectors can be expressed as $R \mathbf{a}-\mathbf{v}$ and $R \mathbf{v}+\mathbf{a}$ for suitable $\mathbf{a}$ and $\mathbf{v}$, this shows that every parity reflected static Galilei transformation is the square of a coset transformation $g_{\Sigma}$. Therefore every connected static Galilei transformation is the fourth power of a coset transformation.

As is well-known, according to Noether's theorem, there exists a conserved quantity corresponding to every continuous symmetry. The conserved quantities for the usual Galilean transformations are standard and will not be repeated here. The conserved quantities for the $S L(2, R)$ symmetry can be derived as follows: The invariance of the Action implies

$$
\delta L(x, \dot{x}, t)=\frac{d \mathcal{F}}{d t}
$$

For time-independent Lagrangians $L\left(x_{i}, \dot{x}_{i}\right)$, we have

$$
\delta L=\frac{\partial L}{\partial x_{i}} \delta x_{i}+\frac{\partial L}{\partial \dot{x}_{i}} \delta \dot{x}_{i}=\frac{d}{d t}\left(\frac{\partial L}{\partial \dot{x}_{i}} \delta x_{i}\right)
$$

where the Euler-Lagrange equations have been used in the second equality. Combining the two expressions for $\delta L$, we get a conservation law

$$
\frac{d}{d t}\left(\frac{\partial L}{\partial \dot{x}_{i}} \delta x_{i}-\mathcal{F}\right)=0
$$


The $S L(2, R)$ transformations are

$$
\begin{aligned}
t & \rightarrow \tau=\frac{\alpha t+\beta}{\gamma t+\delta} \\
x(t) & \rightarrow \xi(\tau)=\frac{x(t)}{\gamma t+\delta}=(\alpha-\gamma \tau) x\left(\frac{\delta \tau-\beta}{-\gamma \tau+\alpha}\right)
\end{aligned}
$$

For infinitesimal transformations, $\alpha=1+\varepsilon$ and $\delta=1-\varepsilon$ (to ensure $\alpha \delta-\beta \gamma=1$ ) with infinitesimal $\beta, \gamma, \varepsilon$,

$$
\begin{aligned}
\delta t & =\beta+2 \varepsilon t-\gamma t^{2} \\
\delta x(t) & =(\varepsilon-\gamma t) x(t)-\left(\beta+2 \varepsilon t-\gamma t^{2}\right) \dot{x}(t)
\end{aligned}
$$

The change of $L=(m / 2) \dot{x}^{2}$ is given by

$$
\delta L=m \dot{x} \cdot \delta \dot{x}=m \dot{x} \cdot\left(-\gamma x-(\varepsilon-\gamma t) \dot{x}-\left(\beta+2 \varepsilon t-\gamma t^{2}\right) \ddot{x}\right)
$$

It is easily seen to be the total time derivative of

$$
\mathcal{F}=-\left(\beta+2 \varepsilon t-\gamma t^{2}\right) \frac{m \dot{x}^{2}}{2}-\gamma \frac{m x^{2}}{2}
$$

Therefore, we obtain the conserved quantity

$$
X=m \dot{x} \cdot \delta x-\mathcal{F}=-\left(\beta+2 \varepsilon t-\gamma t^{2}\right) \frac{m \dot{x}^{2}}{2}+(\varepsilon-\gamma t) m x \cdot \dot{x}+\gamma \frac{m x^{2}}{2}
$$

Extracting the coefficients of $\beta, \varepsilon$ and $\gamma$ we get

$$
\begin{aligned}
X & =-\beta H+\varepsilon D+\gamma A \\
H & =\frac{p^{2}}{2 m} \\
D & =p \cdot\left(x-\frac{t p}{m}\right) \\
A & =\frac{(t p-m x)^{2}}{2 m}
\end{aligned}
$$

where $p=m \dot{x}$ and $H, D, A$ are the conserved quantities related to time translations, dilatations, and expansions respectively. The following interesting observation about the 
conserved quantities can now be made: The Noether's theorem can also be used to show that the conserved quantities corresponding to the usual translation and boost symmetries are $p_{i}=m \dot{x}_{i}$ and $K_{i}=t p_{i}-m x_{i}$ respectively; hence it follows that $A=K^{2} / 2 m$ is related to $K$ in the same way as the Hamiltonian is related to the momenta. Mathematically, $H, A$ and $D=-p \cdot K / m$ form the adjoint representation of the $S L(2, R)$, while $p$ and $K$ transform as a doublet, and rotations are invariant. This concludes the discussion of the symmetries of the classical non-relativistic point particle.

We shall now consider the quantum mechanical generalisation of the above results. For this it is convenient to think of the wavefunction of the particle as a non-relativistic field. For the usual ten-parameter Galilei group $G$, it is well-known that, in the field theoretic realisation, there is a one-parameter mass group $M$ that commutes with $G$. This is called the central extension and has the effect of modifying the Lie algebra of $G$ in a non-trivial manner, while at the same time preserving the Jacobi identity. It turns out that a similar feature holds for the twelve-parameter group $\mathcal{G}$.

In the field representation the conserved quantities of $\mathcal{G}$ are

$$
\begin{gathered}
\mathbf{P}=-i \nabla, \quad \mathbf{J}=-i \mathbf{x} \times \nabla \quad \mathbf{K}=-i t \nabla-m \mathbf{x} \\
D=i\left(2 t \frac{\partial}{\partial t}+\mathbf{x} \cdot \nabla+\frac{3}{2}\right), \quad A=-i\left(t^{2} \frac{\partial}{\partial t}-\frac{m}{2} \mathbf{x}^{2}+t \mathbf{x} \cdot \nabla+\frac{3}{2} t\right), \quad H=i \frac{\partial}{\partial t}
\end{gathered}
$$

where the factors of $3 / 2$ appear because we have Weyl ordered products of position and momentum in the quantum theory and set $\hbar=1 . \mathbf{P}, \mathbf{J}, \mathbf{K}$ generate translations, rotations and boosts respectively, which constitute the connected static Galilei group (21). $H, D$, and $A$ produce time translations, dilations, and expansions which together constitute the $S L(2, R)$ group. The central extension of the standard Lie algebra of the former

$$
\begin{gathered}
{\left[J_{i}, J_{k}\right]=i \epsilon_{i k l} J_{l},\left[J_{i}, P_{k}\right]=i \epsilon_{i k l} P_{l}, \quad\left[P_{i}, P_{k}\right]=0} \\
{\left[K_{i}, K_{k}\right]=0,\left[J_{i}, K_{k}\right]=i \epsilon_{i k r} K_{r},\left[K_{i}, P_{k}\right]=-\left(i m \delta_{i k}\right)}
\end{gathered}
$$




$$
\left[P_{i}, H\right]=0 \quad\left[K_{i}, H\right]=-i P_{i}, \quad\left[J_{i}, H\right]=0
$$

is augmented by the additional relations involving the $S L(2, R)$ generators

$$
\begin{gathered}
{\left[D, J_{i}\right]=0, \quad\left[D, K_{i}\right]=i K_{i}, \quad\left[D, P_{i}\right]=-i P_{i}} \\
{\left[A, J_{i}\right]=0, \quad\left[A, K_{i}\right]=0, \quad\left[A, P_{i}\right]=i K_{i}} \\
{[D, H]=-2 i H, \quad[A, H]=i D, \quad[D, A]=2 i A}
\end{gathered}
$$

to give the Lie algebra of the full group $\mathcal{G}$. The bracketed term in the $\left[K_{i}, P_{j}\right]$ commutator is the non-trivial modification that the central extension brings about in the Lie algebra with $m$ - to be physically identified with the mass of the particle - standing for the value that the generator of $M$ takes in the given representation.

Since the existence of the central extension implies that

$$
\left[K_{i}, P_{j}\right]=-\left(i m \delta_{i j}\right)
$$

the groups of translations $T(\mathbf{a})=e^{i \mathbf{a} \cdot \mathbf{P}}$ and boosts $B(\mathbf{v})=e^{i \mathbf{v} \cdot \mathbf{K}}$ no longer form a direct product, but a Heisenberg-Weyl group defined by the relation

$$
T(\mathbf{a}) B(\mathbf{v})=B(\mathbf{v}) T(\mathbf{a}) e^{i m \mathbf{a} \cdot \mathbf{v}}
$$

The action (24) of $S L(2, R)$ on $\mathbf{a}$ and $\mathbf{v}$ induces the transformations

$$
\sigma^{-1}\left(\begin{array}{l}
\mathbf{K} \\
\mathbf{P}
\end{array}\right) \sigma=\left(\begin{array}{ll}
\alpha & \beta \\
\gamma & \delta
\end{array}\right)\left(\begin{array}{l}
\mathbf{K} \\
\mathbf{P}
\end{array}\right)
$$

of the generators $\mathbf{K}$ and $\mathbf{P}$. The commutator (40) does not change under these transformations because of the condition $\alpha \delta-\beta \gamma=1$. Thus the central extension is compatible with $S L(2, R)$ and the full invariance group of the quantised system is the central extension of $\mathcal{G}$.

Indeed it was in the quantum theory that the group $\mathcal{G}$ was first discovered by Niederer who showed that it is the maximal kinematical invariance group of the free 
particle Schrödinger equation [4]. Since the invariance under the Galilei group is wellknown, it suffices to verify that the Schrödinger equation

$$
i \frac{\partial \psi}{\partial t}+\frac{1}{2 m} \frac{\partial^{2} \psi}{\partial x_{i}^{2}}=0
$$

remains form-invariant under $S L(2, R)$. It is easily checked that this is accomplished by the following transformation of the wavefunction that is generated by $A, H$, and $D$ of the $S L(2, R)$ group.

$$
\psi(\mathbf{x}, t) \propto(\gamma \tau-\alpha)^{\frac{3}{2}} e^{i \frac{F}{2}} \psi(\xi, \tau)
$$

where $F$ is determined through (8) and (9).

As promised, we now sketch a road map to an astrophysical application of the results of this paper. As is explained in detail in [2], recent experimental programmes try to simulate astrophysical systems like supernova explosions in the laboratory by creating implosions in inertial confinement plasmas. This research is inspired by a remarkable similarity which was observed in the results of numerical models of 1987A supernova observations and results of numerical simulations of experiments involving plasma implosions. Referring the reader to [2] for further details of this research programme, we note that this is a puzzling observation because the former system involves very large length and time scales whereas the latter involves very small scales. A theoretical explanation of this intriguing similarity was given in [1] and can be traced to the symmetry properties of the fluid dynamic equations $[5,6]$ that describe both stellar structure and the plasma state. As conjectured in [1], this symmetry has its origin in the symmetries of the free point particle. In particular the symmetry responsible for mapping supernova explosions to plasma implosions is the fluid mechanical analogue of the expansion transformations discussed in this paper. To make this connection more precise, we note that the analysis of the single free point particle can be carried over to 
the non-interacting many particle case in a straightforward manner. Indeed, using the expression for the Hamiltonian of an ensemble of free point particles labelled by $I$, and the expression for the momenta $\mathbf{p}_{I}$,

$$
H=\sum_{I} \frac{\mathbf{p}_{I}^{2}}{2 m} \quad \text { and } \quad \mathbf{p}_{I}=m \dot{\mathbf{x}}_{I}
$$

it is easy to see that the corresponding Liouville equation stating the invariance of the density of particles $\rho$ along the flow

$$
\frac{d \rho}{d t}=\frac{\partial \rho}{\partial t}+\{\rho, H\} \quad=0
$$

also has a maximal invariance group given by (26). As is well-known, the Liouville equation can be converted into the Boltzmann equation by expressing all the momenta in terms of the velocities. Therefore, the symmetry group carries over to the collisionless Boltzmann equation. Further, in the continuum limit, one can use the standard procedure of deriving the various fluid dynamic equations as moments of the Boltzmann equation[7]. The simplest example is the set of Euler equations [8]

$$
\begin{gathered}
D \rho=-\rho \nabla \cdot \mathbf{u} \\
\rho D \mathbf{u}=-\nabla p \\
D \epsilon=-(\epsilon+p) \nabla \cdot \mathbf{u}
\end{gathered}
$$

where $\rho, \mathbf{u}, p$ and $\epsilon$ stand for the density, the velocity vector field, the pressure, and the energy density of the fluid respectively. The convective derivative $D$ in the above equations is defined by $D=\frac{\partial}{\partial t}+\mathbf{u} \cdot \nabla$. The above differential equations of fluid flow are usually augmented by an algebraic condition called the polytropic equation of state which relates the pressure to the energy density thus:

$$
p=\left(\gamma_{o}-1\right) \epsilon
$$


where $\gamma_{o}$ is a constant called the polytropic exponent. For the ensemble of free nonrelativistic point particles being considered here, $\gamma_{0}$ takes the value $5 / 3$. The maximal symmetry group $\mathcal{G}$ therefore extends to the fluid dynamic equations which explains the observed similarity between supernova explosions and plasma implosions.

Although we have considered free particles so far, it is interesting to note that the symmetry group discussed in this paper extends to an interesting class of interacting many-body problems namely, those for which the potential is an inverse square of the coordinate differences. In one dimension these include the so-called Calogero-Moser models [9] with Hamiltonian

$$
H=\frac{1}{2}\left(\sum_{I=1}^{N} p_{I}^{2}+g^{2} \sum_{I \neq J} \frac{1}{\left(x_{I}-x_{J}\right)^{2}}\right)
$$

These models are integrable and admit exclusion statistics - an exciting area of current research [10]. In two dimensions it may be shown that models with Hamiltonians of the form

$$
H=\frac{1}{2}\left(\sum_{I=1}^{N}\left(\mathbf{p}_{I}-e \mathbf{A}\left(\mathbf{x}_{I}\right)\right)^{2}\right), \quad \text { where } \quad A_{k}\left(\mathbf{x}_{I}\right) \propto \sum_{J \neq I} \frac{\epsilon_{k l}\left(\mathbf{x}_{I}-\mathbf{x}_{J}\right)_{l}}{\left|x_{I}-x_{J}\right|^{2}}
$$

which describe a gas of anyons - particles having arbitrary spin and statistics - have potentials which admit the symmetry group $\mathcal{G}$. As is well-known, anyons are of interest because they appear as excitations in fractional quantum Hall systems [11].

To conclude, we have investigated the maximal kinematical invariance group of a free nonrelativistic point particle and have found that it is bigger than the Galilei group. It is a semi-direct product of the form $S L(2, R) \wedge G$ where $G$ is the static Galilei group. As shown in this paper, this group is in fact the maximal invariance group of a host of interesting systems in which the physics content is captured by the quintessential free nonrelativistic point particle. However there exists a class of interacting many particle systems - of which the well-known Calogero-Moser models and the anyon model are of particular interest - for which this is also true. 


\section{References}

1. L. O'C Drury and J. T. Mendonça, "Explosion implosion duality and the laboratory simulation of astrophysical systems," Physics of Plasmas, 7 No.12 (2000) 5148-5142.

2. Supernova Hydrodynamics Up Close: Science and Technology Review, Jan'/Feb' 2000; http://www.Ilnl.gov/str; I. Hachisu et al., "Rayleigh-Taylor instabilities and mixing in the helium star models for Type Ib/Ic supernovae," Astrophysical Journal 368 (1991) L27-30; H. Sakagami and K. Nishihara, "Rayleigh-Taylor instability on the pusher-fuel contact surface of stagnating targets," Physics of Fluids B 2 (1990) $2715-2730$.

3. P. J. Olver, Applications of Lie Groups to Differential Equations; Springer-Verlag (1986).

4. U. Niederer, "The maximal kinematical invariance group of the free Schrödinger equation," Helvetica Physica Acta, 45 (1972) 802-810.

5. L. O'Raifeartaigh and V. V. Sreedhar, "The maximal kinematical invariance group of fluid dynamics and explosion-implosion duality," hep-th/0007199.

6. M. Hassaine and P. A. Horvathy, "Field-dependent symmetries of a non-relativistic fluid model," math-ph/9904022, "Symmetries of fluid dynamics with polytropic exponent," Phys. Lett. A279 (2001) 215-222; S. Deser, R. Jackiw and A. P. Polychronakos, "Clebsch (string) parameterization of 3-vectors and their actions," physics/0006056; R. Jackiw, V. P. Nair and So-Young Pi, "Chern-Simons reduction and non-Abelian fluid mechanics," Physical Review D 62 (2000) 085018, hepth/0004084; R. Jackiw, "A particle field theorist's lectures on supersymmetric, non-Abelian fluid mechanics and D-branes," physics/0010042.

7. K. Huang, Statistical Mechanics; John Wiley and Sons (1967).

8. L. D. Landau and E. M. Lifshitz, Fluid Mechanics, Pergamon Press (1959).

9. F. Calogero, "Exactly solvable one-dimensional many body problems," Lett. Nuov. 
Cim. 13 (1975) 411; J. Moser, "Three integrable Hamiltonian systems connected with isospectral deformations," Adv. Math. 16 (1975) 197-220.

10. A. Polychronakos, "Generalized statistics in one dimension," hep-th/9902157; Lectures at the Les Houches Summer School in Theoretical Physics, Session 69: Topological Aspects of Low-dimensional Systems.

11. F. Wilczek, Fractional Statistics and Anyon Superconductivity; World Scientific (1990). 